\title{
Designed proteinoid polymers and nanoparticles encapsulating risperidone for enhanced antipsychotic activity
}

\author{
L. Lugasi' , I. Grinberg' ', S. Rudnick-Glick', E. Okun², H. Einat ${ }^{3}$ and S. Margel ${ }^{1 *}$ (I)
}

\begin{abstract}
Background: Nanoparticles (NPs) incorporating drug formulations can be used to facilitate passage through biological barriers including the blood-brain barrier (BBB) and increase drug delivery and bioavailability. Hence, NP-based administration may enhance the efficiency of current antipsychotics. Encapsulation within NPs can resolve aqueous solubility problems that not only reduce permeability through the BBB but also affect targeting. The present study describes a new drug delivery system based on proteinoid NPs to explore the possibility of improving drug efficacy. Risperidone (RSP) is a commonly used atypical antipsychotic medication, and was therefore selected for encapsulation by proteinoid NPs.

Results: Proteinoid polymers with high molecular weight and low polydispersity were synthesized from L-amino acids and poly-L-lactic acid (PLLA) by thermal step-growth polymerization mechanism. RSP-loaded proteinoid NPs were then prepared using a self-assembly process in the presence of RSP, followed by PEGylation. The optimal PEGylated RSP-loaded NPs were characterized in terms of diameter and size distribution, drug loading, $\zeta$-potential, cytotoxicity, biodistribution, and psychopharmacological effects. The findings indicate significantly higher antipsychotic activity of drug-loaded proteinoid NPs compared to free RSP.
\end{abstract}

Conclusions: Proteinoid NPs enhance RSP delivery and may potentially increase drug efficiency by reducing dosage and side effects.

Keywords: Proteinoid nanoparticles, Self-assembly, Risperidone, Drug delivery, Antipsychotic therapy

\section{Background}

Mental disorders are cognitive behavioral or emotional patterns that cause significant distress and/or permanent impairment of personal functions [1]. They affect approximately $13 \%$ of the global population. Signs and symptoms vary, ranging from mild to moderate and even severe impairment. Triggers of mental disorders include genetics [2], biochemical processes in brain structure and development [3], environmental changes

*Correspondence: Shlomomargel@biu.ac.i

${ }^{1}$ Department of Chemistry, The Institute of Nanotechnology and Advanced Materials, Bar-Ilan University, 5290002 Ramat Gan, Israel

Full list of author information is available at the end of the article
[4], traumatic life events [5], drug use, and lifestyle [6]. Treatment is disorder- and individual-specific and usually combines psychotherapy and medication.

Antipsychotics (also called neuroleptics or major tranquilizers) are a class of medications that are primarily used in the treatment of psychoses, in particular schizophrenia and bipolar disorders [7]. Risperidone (RSP, Fig. 1) is considered as one of the most reliable and effective atypical (second generation) antipsychotic medications, and is widely used in the treatment of irritability associated with autism and both positive (e.g., hallucinations or delusions) and negative (e.g., emotional withdrawal or loss of speech) symptoms of schizophrenia and bipolar disorders [8-10]. Schizophrenia 
and bipolar disorders are progressive illnesses with an incidence of approximately $1-2.5 \%$ of the population.

Risperidone, 3-\{2-[4-(6-fluoro-1,2-benzisoxazol3-yl)-1-pip-eridin] ethyl\}-6,7,8,9-tetrahydro-2-methyl$4 \mathrm{H}$-pyrido-[1,2-a]pyrimidine-4-one, is a benzoisoxazole derivative with strong binding affinity for serotonin 5-HT2 and dopamine D2 receptors, as well as high affinity to $\alpha_{1}$ - and $\alpha_{2}$-adrenergic receptors and histamine $\mathrm{H}_{1}$ receptors [11, 12]. In humans, RSP undergoes extensive metabolism mainly by hydroxylation and oxidative $\mathrm{N}$-dealkylation to a 9-hydroxyrisperidone metabolite that is subjected to generic polymorphism [13]. Recent studies have shown that polymorphism of the CYP2D6 gene plays an important role in RSP metabolism by the enzyme expressed in the blood, and indicate that RSP is a highly unstable molecule and a poorly water-soluble weak base, which belongs to Class II of the Biopharmaceutics Classification System. This leads to erratic bioavailability requiring frequent dose revision [14].

Atypical antipsychotics are less likely to cause extrapyramidal side effects. Although RSP is less sedating than other atypical antipsychotics, it tends to have sexual side effects and after-effects of increasing the risk of weight gain and metabolic problems, and is likely to have more extrapyramidal side effects $[12,15]$. Therefore, there is a need to find effective formulations of these hydrophobic therapeutic compounds to improve solubility, stability and drug efficiency and reduce side effects.

Biodegradable NPs have gained importance in the field of nanomedicine as they are able to improve targeted drug delivery, biocompatibility, bioavailability, safety, permeability and retention time and reduce toxicity [16-21]. Formulation-based biodegradable NPs are efficient vehicles for drug transport to targeted sites. Previous studies demonstrated that precisely designed biodegradable NP carriers can cross the blood brain barrier (BBB), improve administration, and control drug targeting [20-24].

Proteinoids are random polymers consisting of amino acids, synthesized by thermal step-growth polymerization [25-30]. These polymers are biodegradable and resemble natural proteins, thus expressing non-immunogenic and non-toxic characteristics [31-33]. One notable advantage of proteinoids as synthetic polymers is that they can be specifically designed to suit a wide range of properties in biomedical applications, including drug delivery systems [34].

In an aqueous solution, by means of a self-assembly mechanism, proteinoids can fold to form hollow particles [35]. The self-assembly process is made possible by the presence of the many functional groups that form part of the random polymer backbone. In an aqueous solution, the hydrophobic residues of the proteinoid are assembled to form a hydrophobic core within the particle matrix to minimize their contact with the aqueous continuous phase, while the hydrophilic residues are exposed to form hydrogen bonds on the particle surface $[36,37]$. The hollow interior of the particles, which is formed during self-assembly, can be utilized to encapsulate various molecules, making proteinoids NPs biocompatible drug carriers [38]. Earlier studies by our group demonstrated that encapsulation of therapeutics such as anti-cancer drugs and near infrared (NIR) fluorescent dyes have potential use in cancer diagnosis and therapy [32,39,40].

In the present study, a series of proteinoids with high molecular weight and low polydispersity were designed from L-amino acids in the presence and absence of poly(L-lactic acid) (PLLA). PLLA was incorporated within the copolymer backbone to enhance the biodegradability and elicit mechanical rigidity [41-43].

High drug loading (DL) capacity and prolonged blood stream circulation are essential requirements for drug carriers [44]. Polyethylene glycol (PEG) coating (PEGylation) shields the NP surface from aggregation and phagocytosis and thereby impacts NPs administration; it prolongs the retention time in the blood stream, improves delivery of the therapeutic cargo, enhances passage through the $\mathrm{BBB}$, and facilitates distribution and clearance from the body [45-49].

Encapsulation of RSP in nano-carrier delivery systems such as biodegradable NPs was hypothesized to stabilize it; along with drug dosage tailoring, such encapsulation may enhance the tolerability and adherence and increase the bioavailability, thereby improving the antipsychotic activity and reducing the side effects. For this purpose, a psychostimulant-induced behavioral model, which is frequently used to model facets of schizophrenia and mania, especially in the context of screening potential novel treatments, was carried out. Whereas a number of psychostimulants are used in this context, the more established drug is amphetamine [50]. Specifically, in the amphetamine-induced hyperactivity model, single or multiple doses of amphetamine are administered to a model animal at appropriate doses that induce hyperactivity (including increased movement during a testing session) as well as other behavioral changes. Antipsychotic drugs, including RSP [8] and other mood stabilizers [51] were repeatedly demonstrated to ameliorate

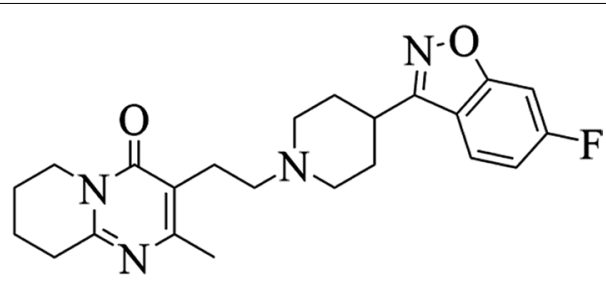

Fig. 1 Chemical structure of risperidone (RSP) 
amphetamine-induced behavioral changes. Accordingly, this model is accepted as a screening model for antipsychotic effects [52]. Here, several PEGylated RSP-loaded proteinoid NPs (Prot./RSP) were introduced in order to increase the stability and solubility of the drug in the aqueous continuous phase, as well as to improve targeting to the brain.

\section{Results}

\section{Design and characterization of proteinoids}

Four different proteinoid polymers were synthesized from Lys or Glu, together with Phe and His, in absence or presence of PLLA, as described in the methods section (see Table 4). The molecular weight $(\mathrm{Mw})$ and polydispersity index (PDI) were determined by gel permeation chromatography (GPC, see Table 1). The basic proteinoids, Prot.1 and Prot.3, exhibited Mw of 168.0 and $154.2 \mathrm{kDa}$, respectively, with similar PDI of 1.00 , whereas the acidic proteinoids, Prot. 2 and Prot. 4 had Mw of 142.6 and $132.3 \mathrm{kDa}$ with PDI of 1.02 and 1.05 , respectively. Prot.1-4 exhibited optical activity of $-11.6,-6.5,7.8$ and 4.8 , respectively.

\section{Formation of hollow and RSP-loaded proteinoid NPs}

Proteinoids were self-assembled to form proteinoid NPs, followed by PEGylation process. The PEGylated hollow NPs (Prot.1-4) in water as a continuous phase had diameters of less than $95 \mathrm{~nm}$ with a narrow size distribution (SD) of $2-7 \%$, in accord with the low PDI of the proteinoid polymers. The hollow NPs, Prot.3 and Prot.4, prepared in the absence of PLLA, yielded diameters of $94 \pm 7$ and $90 \pm 4 \mathrm{~nm}$, respectively, while Prot. 1 and Prot. 2 that incorporate PLLA were smaller with diameters of $78 \pm 4$ and $58 \pm 2 \mathrm{~nm}$, respectively. The RSPloaded NPs (Prot.1-4/RSP NPs) with $20 \% \mathrm{w} / \mathrm{w}$ RSP had a slightly higher diameter range of $86 \pm 3$ to $122 \pm 6 \mathrm{~nm}$, with a similar narrow SD of 2-6\%.

The hydrodynamic diameters of the hollow Prot.1 and Prot. 2 and their corresponding RSP-loaded NPs, Prot.1/ RSP and Prot.2/RSP, dispersed in aqueous continuous phase were analyzed by dynamic light scattering (DLS) and cryogenic transmission electron microscopy (cryoTEM), as shown in Fig. 2 and Table 2. Prot.1 exhibited a population of uniform spherical hollow NPs with diameter of $78 \pm 4 \mathrm{~nm}$ by DLS as shown in the histogram (Fig. 2a and Table 2) and similar diameter of $75 \pm 8 \mathrm{~nm}$ by cryo-TEM (Fig. 2b). Prot.1/RSP produced uniform spherical NPs with higher diameter of $97 \pm 2 \mathrm{~nm}$ by DLS (Fig. 2c and Table 2) and $93 \pm 6 \mathrm{~nm}$ by cryo-TEM (Fig. 2d). Prot.2 was smaller than Prot.1 and yielded a population of uniform spherical hollow NPs with a diameter of $58 \pm 2 \mathrm{~nm}$ by DLS (Fig. 2e and Table 2) and $58 \pm 3 \mathrm{~nm}$ by cryo-TEM (Fig. 2f). Prot.2/RSP also presented smaller diameter compared to Prot.1/RSP of $86 \pm 3 \mathrm{~nm}$ (DLS, Fig. $2 \mathrm{~g}$ and Table 2) and $82 \pm 2 \mathrm{~nm}$ (cryo-TEM, Fig. 2h). These results show that both methods for measuring NP diameter provide similar results; commonly, the diameters measured from the cryo-TEM were the same or slightly lower (by up to $5 \%$ ) than those obtained by the DLS. The dry diameters of Prot.1/RSP and Prot.2/RSP were measured by scanning electron microscopy (SEM, Fig. 3), yielding $74 \pm 4$ and $55 \pm 5 \mathrm{~nm}$, respectively.

The $\zeta$-potentials at $\mathrm{pH}=7.4$ (Table 3 ) were $12 \pm 3$ and $12 \pm 1 \mathrm{mV}$ for the basic hollow proteinoids NPs, Prot.1 and Prot.3, respectively, while the corresponding values for the acidic proteinoids NPs were slightly lower, $-10 \pm 4$ and $-8 \pm 2 \mathrm{mV}$ for Prot. 2 and Prot.4, respectively. The RSP-loaded NPs (Prot.1-4/RSP) exhibited higher negative or positive $\zeta$-potential values of $13 \pm 3$, $-15 \pm 1,13 \pm 3$ and $-12 \pm 2 \mathrm{mV}$.

The DL capacity of Prot.1-4 was determined by high performance liquid chromatography (HPLC) using standard calibration solutions of RSP [7]. The drug was dissolved in DMSO (1\% relative to the total aqueous dispersion volume) to $20 \% \mathrm{w} / \mathrm{w}$ compared to the total proteinoid weight. Table 2 presents the DL (w/w \%) of Prot. 1-4/RSP NPs, $20 \pm 0.1,20 \pm 0.1,19 \pm 0.4$ and $19 \pm 0.3 \%$, respectively. As shown in the table, Prot. 1 and Prot.2 offer better RSP encapsulation owing to the significantly smaller diameters of $78 \pm 4$ and $58 \pm 2 \mathrm{~nm}$, respectively (compared to $94 \pm 7$ and $90 \pm 4 \mathrm{~nm}$ for Prot. 3 and Prot.4, respectively). Prot. 2 also has greater physical stability as indicated by the higher $\zeta$-potential of $-10 \pm 4$, compared to $-8 \pm 2 \mathrm{mV}$ for Prot.4. In addition, Prot. 1 and Prot. 2 also show slightly higher RSP DL of $20 \pm 0.1 \% \mathrm{w} / \mathrm{w}$ compared to $19 \pm 0.3 / 0.4 \% \mathrm{w} / \mathrm{w}$ for Prot. 3,4 .

Table 3 presents an optimization study of PEGylated Prot.1/RSP and Prot.2/RSP NPs. Encapsulation of RSP at 10, 20, 30, 40 and $50 \% \mathrm{w} / \mathrm{w}$ within Prot. 1 formed larger particle diameters of $68 \pm 2,97 \pm 4,143 \pm 5$, $178 \pm 8$ and $215 \pm 7 \mathrm{~nm}$ compared with $68 \pm 2,86 \pm 3$, $136 \pm 5,156 \pm 8$ and $194 \pm 9 \mathrm{~nm}$ for Prot.2. These results indicate that $20 \%$ RSP yields an optimal diameter of $68 \pm 2$ for both Prot.1/RSP and Prot.2/RSP NPs. Higher RSP concentrations yield particles with diameters above $100 \mathrm{~nm}$, which are less suitable for drug delivery.

\section{In vitro cell viability assay}

XTT-based viability assays in cell culture are used to study changes in the number of cells and their metabolic activity. The assay is based on extracellular reduction of XTT by NADH produced in the mitochondria by transplasma membrane electron transport and an electron 
Table $1 \mathrm{Mw}, \mathrm{Mn}, \mathrm{Mp}, \mathrm{PDI}$ and optical activity of the proteinoids

\begin{tabular}{|c|c|c|c|c|c|}
\hline Proteinoid $^{a}$ & $M w^{b}(k D a)$ & $\mathrm{Mn}^{\mathbf{b}}(\mathrm{kDa})$ & $M p^{b}(k D a)$ & $\mathrm{PDI}^{\mathrm{c}}(\mathrm{kDa})$ & $\begin{array}{l}\text { Optical } \\
\text { activity } \\
{[a]_{D}^{25^{\circ} \mathrm{C}}\left({ }^{\circ}\right)^{d}}\end{array}$ \\
\hline Prot.1 & 168.0 & 166.3 & 156.8 & 1.00 & -11.6 \\
\hline Prot.2 & 142.6 & 139.5 & 135.1 & 1.02 & -6.5 \\
\hline Prot.3 & 154.2 & 153.9 & 149.3 & 1.00 & 7.8 \\
\hline Prot.4 & 132.3 & 126.0 & 126.4 & 1.05 & 4.8 \\
\hline
\end{tabular}

a The basic Prot.1,3 and acidic Prot.2,4 proteinoids were prepared at 140 and $180^{\circ} \mathrm{C}$, respectively

b Molecular weights were measured by GPC, Mp is the molecular mass at the peak

c $\mathrm{PDI}$ is the polydispersity index, given by $\mathrm{Mw} / \mathrm{Mn}$

d Specific optical rotation ( $\mathrm{C}=1$ in $\mathrm{H}_{2} \mathrm{O}$ at $25^{\circ} \mathrm{C}$ )

mediator. The effect of PEGylated hollow and RSPloaded proteinoid NPs on J774A.1 and Neuro- $2 \alpha$ cells was examined by XTT (Fig. 4). Prot. 1 and Prot. 2 as well as Prot1/RSP and Prot.2/RSP NPs are not toxic to both cells, showing viability levels close to $100 \%$, similar to the control group (free RSP).

\section{In vitro cell uptake study}

Cell permeability and fluorescent microscopy images of Neuro- $2 \alpha$ cell of Cy7-cojugated PEGylated hollow and RSP-loaded Prot.1 and Prot.2 were analyzed. The study was carried out using flow cytometry and visualized by confocal microscope. Cell nuclei were stained with Hoechst 33342 [53]. Figure 5 exhibits the intracellular fluorescence of Prot.1, Prot.2, Prot.1/RSP and Prot.2/ RSP. Figure 5a demonstrates fluorescence-activated cell sorting (FACS) analysis of murine Neuro- $2 \alpha$ cell uptake after $4 \mathrm{~h}$ of all four Cy-7 conjugated NPs. Figure $5 \mathrm{~b}$ and c present the intracellular fluorescence of the NPs on Neuro- $2 \alpha$ and show that the NPs penetrate the membrane and are taken up by the cells.

\section{In vivo biodistribution studies}

In order to determine the in vivo body distribution and blood half-life of Cy7-conjugated Prot.1 and Prot.2, $0.2 \mathrm{mg} / \mathrm{mL}$ of each proteinoid NP were injected IV into the tail vein of male BALB/C mice. Blood was drawn at $0,30 \mathrm{~min}, 1 \mathrm{~h}$ and $4 \mathrm{~h}$ post injection. The fluorescence
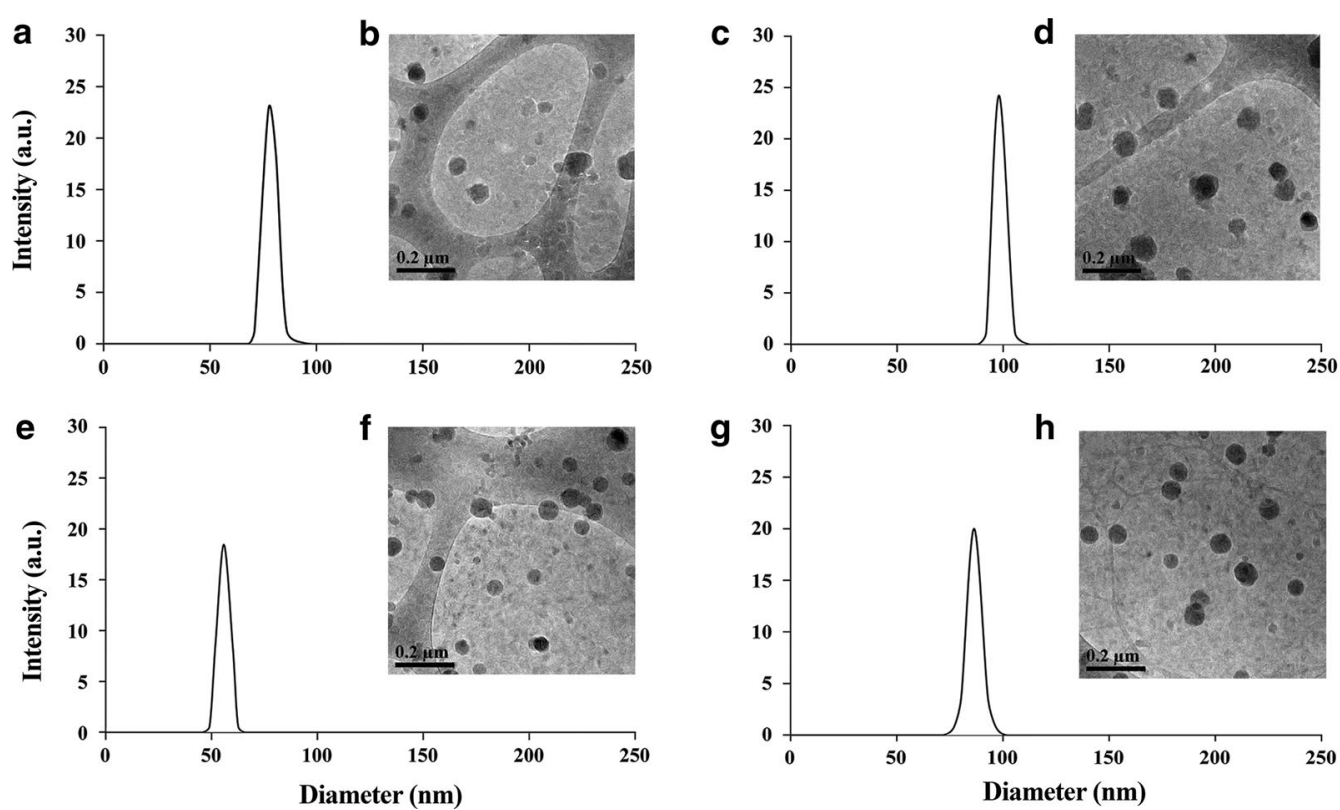

Fig. 2 Diameter histograms by DLS and cryo-TEM images of Prot. $1(\mathbf{a}, \mathbf{b})$, Prot. 1/RSP (c, d), Prot. $2(\mathbf{e}, \mathbf{f})$ and Prot. 2/RSP $(\mathbf{g}, \mathbf{h})$ NPs dispersed in aqueous continuous phase 
Table 2 Hydrodynamic diameter and SD, C-potential and DL of PEGylated proteinoid NPs

\begin{tabular}{lccl}
\hline Proteinoid NPs & Diameter $(\mathbf{n m})$ & ל-potential $(\mathbf{m V})$ & $\mathrm{DL}(\mathbf{w} / \mathbf{w} \%)$ \\
\hline Prot.1 & $78 \pm 4$ & $12 \pm 3$ & - \\
Prot.2 & $58 \pm 2$ & $-10 \pm 4$ & - \\
Prot.3 & $94 \pm 7$ & $12 \pm 1$ & - \\
Prot.4 & $90 \pm 4$ & $-8 \pm 2$ & - \\
Prot.1/RSP & $97 \pm 2$ & $13 \pm 3$ & $20 \pm 0.1$ \\
Prot.2/RSP & $86 \pm 3$ & $-16 \pm 1$ & $20 \pm 0.1$ \\
Prot.3/RSP & $120 \pm 5$ & $13 \pm 3$ & $19 \pm 0.4$ \\
Prot.4/RSP & $122 \pm 6$ & $-12 \pm 2$ & $19 \pm 0.3$ \\
\hline
\end{tabular}

Proteinoid particles were formed by a self-assembly process in aqueous $10 \mu \mathrm{M}$ $\mathrm{NaCl}$ solution containing $1 \%$ DMSO. The initial RSP concentration was $20 \%$ $\mathrm{w} / \mathrm{w}$ relative to the proteinoid. The hollow proteinoids and Prot./RSP NPs were PEGylated. The hydrodynamic diameter was measured by DLS, the $\zeta$-potential was measured at $\mathrm{pH}=7.4$ by a $\zeta$-potential analyzer, and the $\mathrm{DL}$ was measured by HPLC using a calibration curve of standard RSP solutions

decreased over time, and only traces of fluorescence were noticed $4 \mathrm{~h}$ post injection. The blood half-lives of the Cy7-conjugated proteinoid NPs were calculated to be approximately $20 \mathrm{~min}$ (Fig. 6a). Four hours post injection organs were harvested, and a fluorescent signal was obtained from the brain, colon, heart, lungs, spleen, kidneys and duodenum (Fig. 6b).

\section{In vivo psychopharmacological effect}

The amphetamine-induced hyperactivity screening model was used to evaluate the in vivo psychopharmacological effects of RSP-loaded proteinoid NPs. These NPs (Prot.1,2/RSP 20\%) were injected IV immediately before the testing session; saline, free RSP, and hollow NPs served as controls. Exploration-related parameters including distance travelled, time mobile and center time in an open field arena were digitally recorded and analyzed using the ANY-maze software.
Table 3 Hydrodynamic diameter and SD, Z-potential and DL of Prot.1,2/RSP NPs loaded with various concentrations of RSP

\begin{tabular}{lccl}
\hline Prot./RSP (\%) & Diameter (nm) & 弓-potential (mV) & DL (w/w\%) \\
\hline Prot.1/RSP (10\%) & $68 \pm 2$ & $12 \pm 1$ & $10 \pm 0.1$ \\
Prot.1/RSP (20\%) & $97 \pm 4$ & $13 \pm 3$ & $20 \pm 0.1$ \\
Prot.1/RSP (30\%) & $143 \pm 5$ & $17 \pm 2$ & $30 \pm 0.1$ \\
Prot.1/RSP (40\%) & $178 \pm 8$ & $18 \pm 2$ & $40 \pm 0.3$ \\
Prot.1/RSP (50\%) & $215 \pm 7$ & $19 \pm 3$ & $50 \pm 0.2$ \\
Prot.2/RSP (10\%) & $68 \pm 2$ & $-10 \pm 2$ & $10 \pm 0.1$ \\
Prot.2/RSP (20\%) & $86 \pm 3$ & $-16 \pm 1$ & $20 \pm 0.1$ \\
Prot.2/RSP (30\%) & $136 \pm 5$ & $-16 \pm 2$ & $30 \pm 0.3$ \\
Prot.2/RSP (40\%) & $156 \pm 8$ & $-17 \pm 1$ & $40 \pm 0.2$ \\
Prot.2/RSP (50\%) & $194 \pm 9$ & $-19 \pm 3$ & $50 \pm 0.4$
\end{tabular}

Proteinoid particles were formed by a self-assembly process in $10 \mu \mathrm{M} \mathrm{NaCl}$ aqueous solution containing $1 \%$ DMSO. The initial RSP concentration was between 10 and $50 \% \mathrm{w} / \mathrm{w}$ relative to the proteinoid. The hydrodynamic diameter was measured by DLS, the $\zeta$-potential was measured at $\mathrm{pH}=7.4$ by a $\zeta$-potential analyzer, and the DL was measured by HPLC using a calibration curve with standard RSP solutions

As expected, the administration of amphetamine resulted in increased distance travelled, and RSP reduced distance travelled during the session as shown in Fig. 7a. Analysis of variance (ANOVA) indicated significant effect of amphetamine to increase distance $[\mathrm{F}(1,41)=202.1$, $\mathrm{p}<0.001]$, effect of treatment to reduce distance $[\mathrm{F}(5$, $41)=29.3, \mathrm{p}<0.001]$, and interaction $[\mathrm{F}(5,41)=20.3$, $\mathrm{p}<0.001]$. Post-hoc analysis of the first $30 \mathrm{~min}$ of the session (before amphetamine administration) demonstrated that all RSP groups had lower distance compared with saline control $(\mathrm{p}<0.001$; Cohen's $\mathrm{d}>2.5)$. Following amphetamine administration, all RSP groups exhibited reduced activity compared with saline control ( $\mathrm{p}<0.001$; Cohen's $d>2.12$ ). Prot.2/RSP treatment had a stronger effect compared with free RSP $(p=0.043$; Cohen's $\mathrm{d}=1.88)$ with a similar trend for Prot.1/RSP $(\mathrm{p}=0.09$; Cohen's d $=1.73$ ).
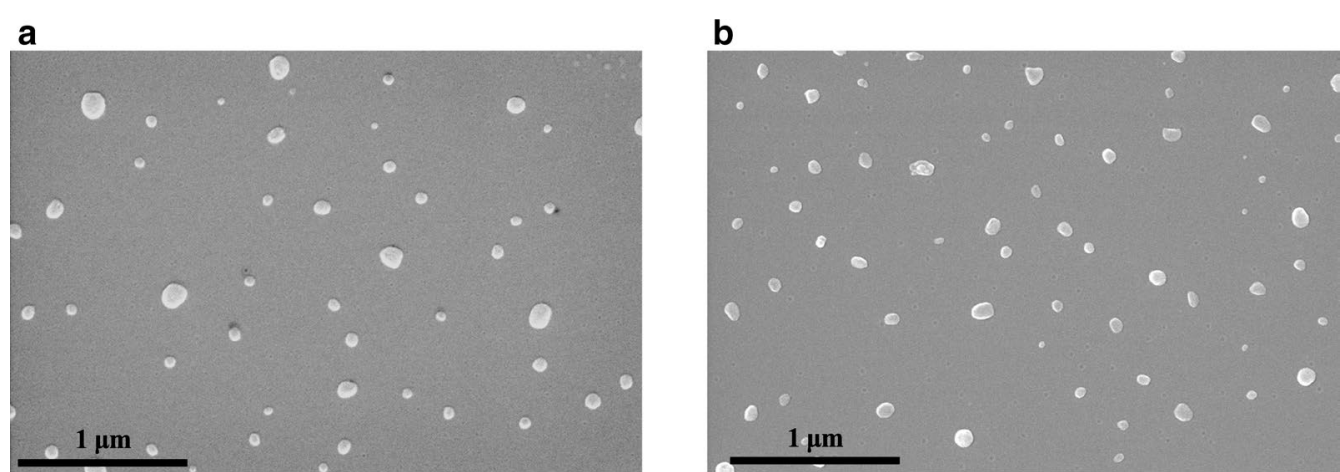

Fig. 3 SEM images of Prot.1/RSP (a) and Prot.2/RSP (b) NPs 
In addition, amphetamine treatment significantly increased time mobile and RSP treatment significantly decreased time mobile (Fig. 7b). A significant effect of amphetamine $[\mathrm{F}(1,41)=13.95, \mathrm{p}=0.001]$ and $\mathrm{RSP}$ treatments $[\mathrm{F}(5,41)=10.82, \mathrm{p}<0.001]$ without interaction $[F(5,41)=1.36, p=0,26]$ were observed using ANOVA. Post-hoc analysis of the first $30 \mathrm{~min}$ of the session demonstrated that mice treated with Prot.1,2/ RSP had lower time mobile compared with saline control group (Prot.1,2/RSP vs. saline $-\mathrm{p} \leq 0.003$, Cohen's $d \geq 1.53$ ). Post-hoc analysis for the second half of the session (after amphetamine administration) showed that treatment with RSP-loaded proteinoid NPs (but not free $\mathrm{RSP}$ ) reduced time mobile compared with saline control $(\mathrm{p} \leq 0.03)$. In addition, the effects of RSP-loaded proteinoids had a trend to reduce time mobile compared with animals treated with free RSP (Prot.1/RSP vs. free

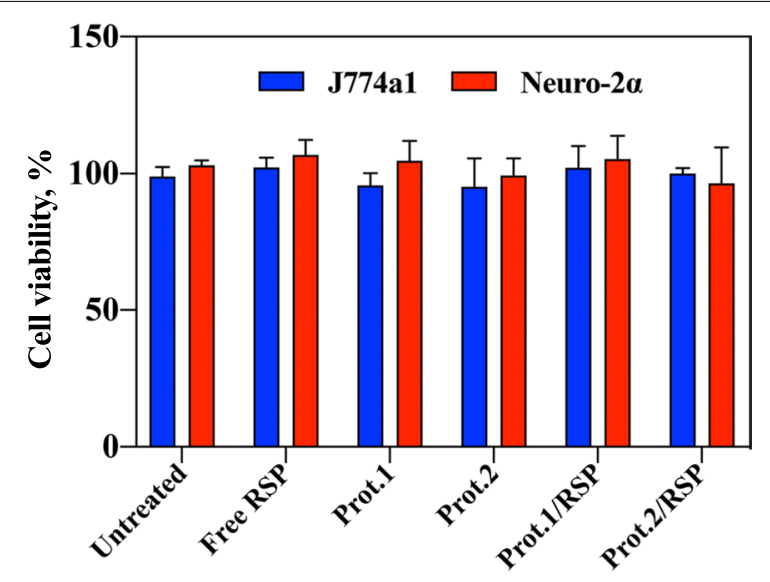

NP treatment

Fig. 4 Cellular mitochondrial metabolic rate by XTT assay in J774A.1 and Neuro-2a cell lines following $48 \mathrm{~h}$ exposure to Prot.1, Prot.2, Prot.1/RSP and Prot.2/RSP PEGylated proteinoid NPs with 10\% PLLA
RSP $-\mathrm{p}=0.055$, Cohen's $\mathrm{d}=1.66$ and Prot.2/RSP vs. free $\mathrm{RSP}-\mathrm{p}=0.1$, Cohen's $\mathrm{d}=1.43$ ).

Time in the center of the open field is considered a measure of anxiety-like behavior [51]. As shown in Fig. 7c, amphetamine administration resulted in decreased time in the center of the arena $[F(1,41)=17.18, p<0.001]$. In contrast, a potent effect to increase time in the center was shown in animals treated with RSP-loaded proteinoid NPs $[(F(5,41)=15.48$, $\mathrm{p}<0.001]$, and there was a significant interaction between the two factors $[\mathrm{F}(5,41)=3.53, \mathrm{p}=0.01]$. The administration of RSP via NPs (both Prot1/RSP and Prot2/RSP) resulted in increased time in the center compared with all other treatments prior to amphetamine treatment $(\mathrm{p} \leq 0.002$; Cohen's $\mathrm{d} \geq 1.7)$ as well as after amphetamine treatment $(\mathrm{p} \leq 0.04$, Cohen's $d \geq 1.39$ ). A heat map analysis of the time occupied by the mice following the different treatments indicated that compared with saline (Fig. $7 \mathrm{~d}_{1,2}$ ), RSP (Fig. $7 \mathrm{e}_{1,2}$ ), Prot.1 (Fig. $7 f_{1,2}$ ), and Prot.2 (Fig. $7 g_{1,2}$ ), both Prot.1/RSP (Fig. $7 h_{1}$, ${ }_{2}$ ) and Prot.2/RSP (Fig. $7 i_{1,2}$ ) groups exhibited significantly reduced activity in the open field arena.

These data support our hypothesis and demonstrate that RSP affects mouse behavior before and after amphetamine administration, with a more pronounced effect after amphetamine treatment. Moreover, the data show that the effects of RSP-encapsulated proteinoid NPs are more pronounced compared with free RSP. These differences are easily visible from the occupancy heat maps and plots produced by the ANY-maze software (Fig. $7 \mathrm{~d}-\mathrm{i}$ ).

\section{Discussion}

Four different proteinoid polymers were synthesized by step-growth polymerization under anhydrous conditions and inert atmosphere $[27,54]$. The proteinoids were synthesized from either Lys or Glu, along with Phe and His, with a weight ratio of $1: 1: 1$ in absence or presence of $10 \%$ w/w PLLA, obtaining two lysine-based and two glutamic
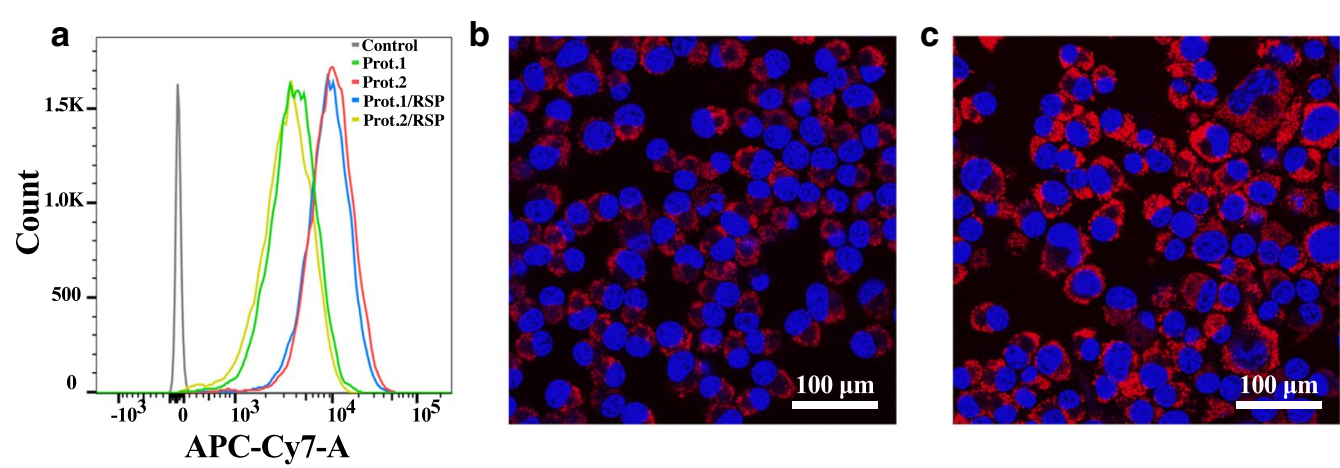

Fig. 5 Intracellular fluorescence of Cy7-conjugated Prot.1 (green), Prot.2 (yellow), Prot.1/RSP (blue) and Prot.2/RSP (red) NPs of Neuro-2a cells by FACS (a). Fluorescence microscopy images of cellular uptake of Cy7-conjugated (cell membrane, red) of Prot.1 (b) and Prot.2 (c) and Hoechst dye (cell nucleus, blue) in Neuro-2a following $24 \mathrm{~h}$ exposure 
acid-based proteinoids (Prot.1-4) as shown in Table 4. Lysine and glutamic acid play a main role in the initiation process, as upon heating, these amino acids undergo homo-cyclization into pyroglutamic acid and caprolactam, respectively (Fig. 8) [55]. They serve as the solvent of the polymerization process and determine the basic and acidic nature of the final proteinoid [56-60]. The additional amino acids, phenylalanine and histidine, were selected for their ability to form $\pi-\pi$ stacking, which enhances the rigidity of the proteinoid. The imidazole group of the histidine facilitates and expedites the proton sponge effect of endosomes, leading to endosomal/ lysosomal escape [61]. PLLA was incorporated within the copolymer backbone to enhance biodegradability via ester functional groups.

Table 1 exhibits the molecular weights and PDI values obtained for the proteinoids by GPC and the optical activity measured by polarimeter. Step-growth polymerization is a random process, hence it is unlikely to obtain high molecular weight with a narrow polydispersity index [62]. In the present study, however, the proteinoids resemble natural proteins, with $\mathrm{Mw}$ in the range of 132 to $168 \mathrm{KDa}$ and low PDI $(1.00-1.05)$ [34, 63]. The chirality of the proteinoids was not affected by the high temperatures used in the synthesis, and all proteinoids remain optically active after the procedure. This is significant and may be utilized in the design of stereospecific drug carriers [64].

Hollow and RSP-loaded proteinoid NPs were prepared, PEGylated, and evaluated in terms of diameter, SD, stability in aqueous continuous phase by $\zeta$-potential, and DL, as shown in Table 2.

As expected, proteinoid NPs containing RSP have larger diameter compared to the corresponding hollow NPs. An increase in size is thus an indication that RSP is indeed entrapped within the proteinoid NPs.

The smaller diameters of Prot.1 and Prot. 2 compared to Prot. 3 and Prot.4 may result from the increased negative charge of the NPs that contain PLLA and the increased rigidity of hydrophobic segments induced by the higher content of phenylalanine and histidine in the interior part of these proteinoid particles.

It should be noted that both methods, DLS and cryoTEM, measure the hydrodynamic diameter and distribution of the NPs, while SEM images measure the dry diameter. Figure 3, for example, illustrates SEM images of the Prot.1/RSP and Prot.2/RSP NPs, which yield significantly smaller dry diameters than the hydrodynamic diameters. Prot.1/RSP and Prot.2/RSP exhibit dry diameters of $74 \pm 4$ and $55 \pm 5 \mathrm{~nm}$, respectively, while the hydrodynamic diameters, as measured by DLS (Table 3 ) were $97 \pm 2$ and $86 \pm 3 \mathrm{~nm}$, respectively. DLS measurements take into consideration the hydrated water


C
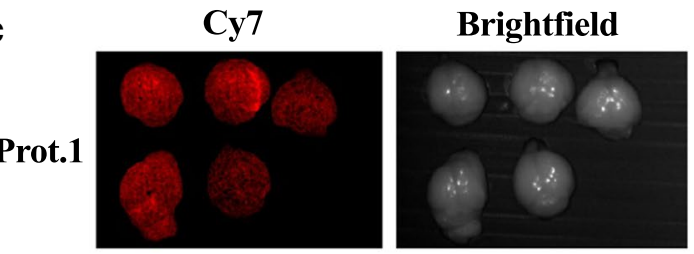

Prot.2
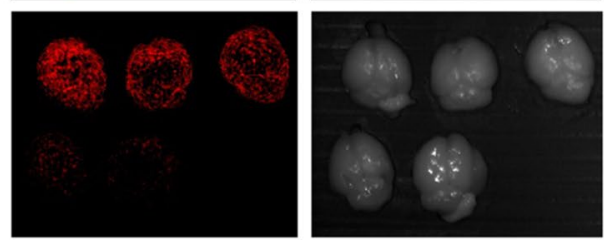

Fig. 6 Biodistribution of proteinoid NPs. (a) Blood clearance of Prot.1,2 NPs. $100 \mu \mathrm{L}$ of Cy7-conjugated Prot.1 and Prot.2 NPs (0.2 mg/ $\mathrm{mL}$ ) were IV injected via the tail vein and blood was drawn at different time intervals, (b) Fluorescence of Cy7-conjugated Prot.1,2 NPs in different organs. Mice were sacrificed $4 \mathrm{~h}$ post injection and organs were harvested. (c) Fluorescent signals and brightfield images of Cy7-conjugated Prot.1,2 NPs using the Maestro in vivo imaging system

layers adsorbed onto the particle surface, which lead to increased diameter relative to the dry measurements.

The physical stability (against agglomeration) of the aqueous dispersions of both PEGylated hollow and RSPloaded proteinoid NPs was studied by $\zeta$-potential $[65,66]$. 


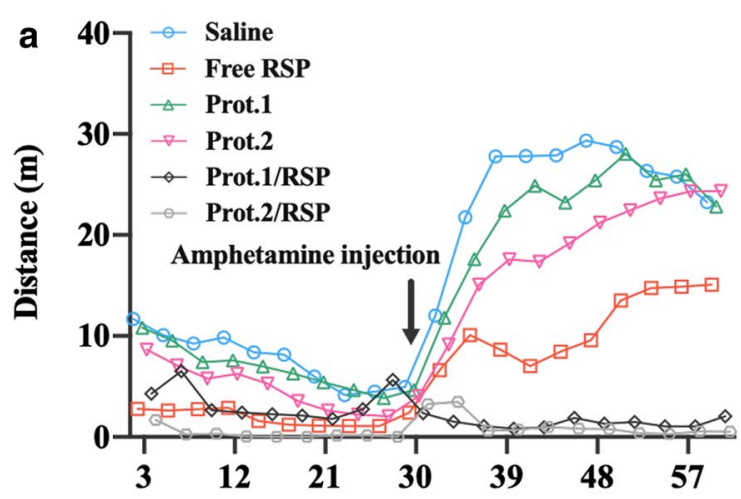

d1
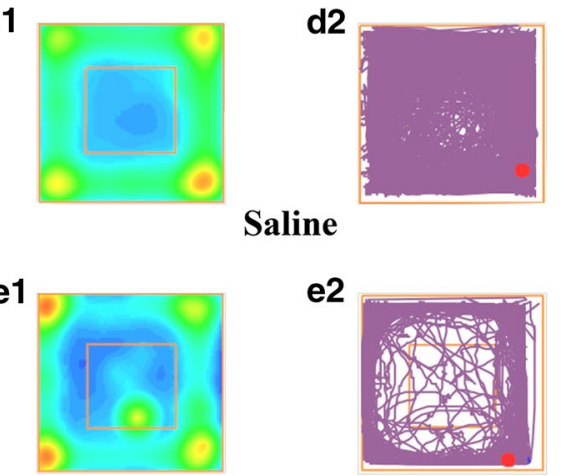

Free RSP
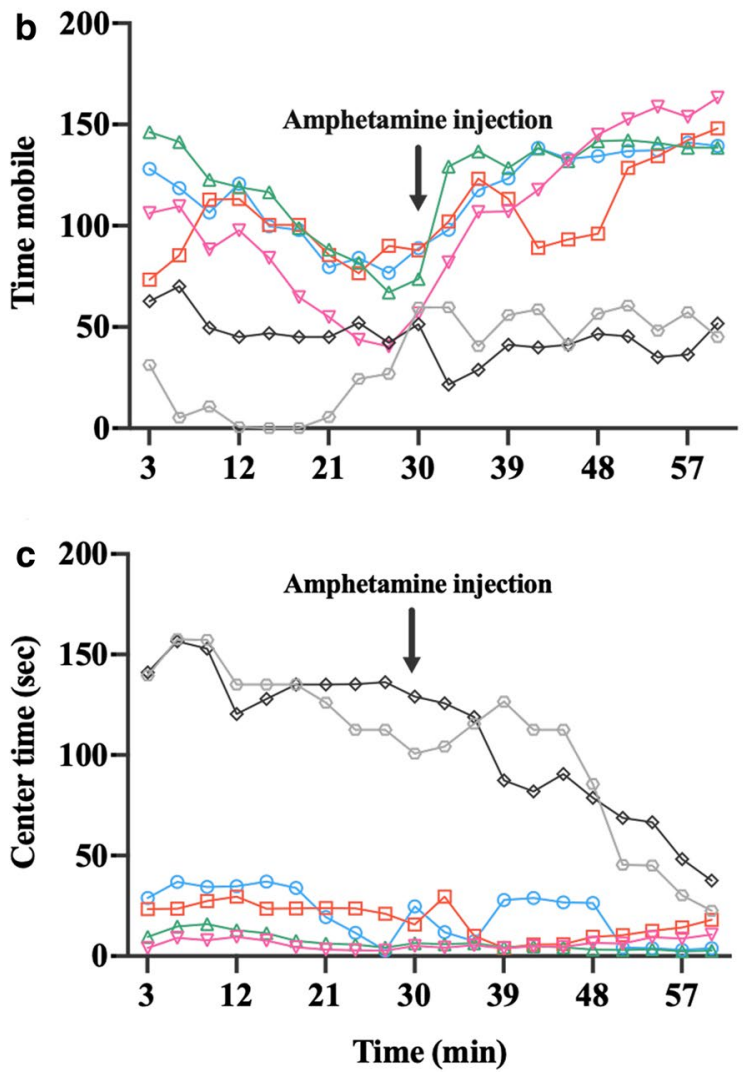

f1

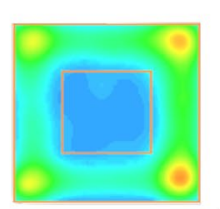

Prot.1

g1

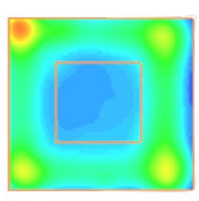

h1

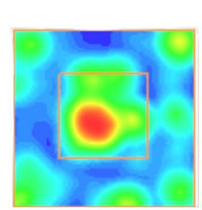

Prot.2

f2

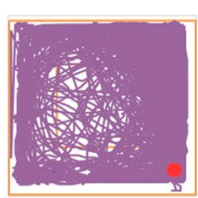

g2

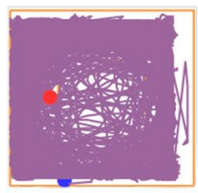

h2

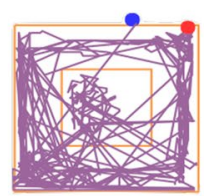

Prot.1/RSP

i1

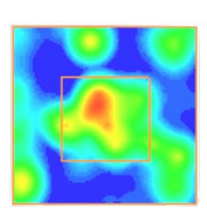

i2

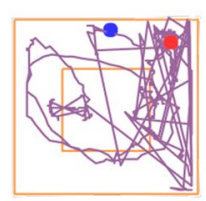

Prot.2/RSP

Fig. 7 Analysis of open field test NPs treatment prior (0-30 min) and post (30-60 min) amphetamine IP uptake using ANOVA. Recorded tracking of amphetamine-induced hyperactivity screening model during 60 min session of distance travelled (a), time mobile (b) and center time (c). Occupancy heat maps and plots produced by the ANY-maze software $\left(\mathbf{d}_{1-2}-\mathbf{i}_{1-2}\right)$

The $\zeta$-potential indicates the degree of repulsion between charged particles in the aqueous dispersion (negative or positive) and the ability to prevent particle aggregation. Table 2 presents the $\zeta$-potentials at $\mathrm{pH}=7.4$ of the hollow and RSP-loaded proteinoid NPs. Prot.1 and Prot.2 are slightly more stable than Prot. 3 and Prot.4, probably due to the added PLLA. The positive $\zeta$-potential values for the basic proteinoid NPs (Prot.1,3) derive from the protonated amine groups on Lys and His, while for the acidic Prot.2,4 the values were negative due to carboxylate groups of Glu that are located on the outer shell of the particles, exposed to the aqueous continuous phase.

Table 3 exhibits the diameter and SD, $\zeta$-potential and DL of the PEGylated Prot.1/RSP and Prot.2/RSP NPs loaded with increasing concentrations of RSP $(10,20,30$, 40 and $50 \% \mathrm{w} / \mathrm{w}$ ). Table 3 illustrates that as the RSP concentration increases, the hydrodynamic diameter of the particles increases. Furthermore, the $\zeta$-potential of the 
particles slightly increases with increasing RSP concentration, which is probably due to successful PEGylation process in which the free amino groups are conjugated to PEG.

Based on these results it can be concluded that PEGylated Prot.1 and Prot.2 NPs without and with 20\% $\mathrm{w} / \mathrm{w}$ RSP are the most suitable particles for continuation of the present study. Their physical stability as measured by $\zeta$-potential and DL is higher than that of the other NPs, and they possess hydrodynamic diameters below $100 \mathrm{~nm}$ and thus have the potential to cross the BBB and target different regions of the brain and enhance the therapeutic effect of RSP.

In addition, leakage of RSP from Prot.1,2/RSP (20\% $\mathrm{w} / \mathrm{w})$ aqueous dispersions $(10 \mathrm{mg} / \mathrm{mL})$ into the aqueous continuous phase was not detected after 3 months of storage at $4{ }^{\circ} \mathrm{C}$, as described in the methods section. Long-term stability against agglomeration of the PEGylated Prot.1,2 and Prot.1,2/RSP $(20 \% \mathrm{w} / \mathrm{w})$ was accomplished by adding trehalose $(10 \mathrm{mg})$ to the NP aqueous dispersions ( $1 \mathrm{~mL}$ containing $10 \mathrm{mg}$ of NPs) followed by lyophilization. The NP powder was redispersed after 1 year in water to the original concentration. The measured diameter and SD of the redispersed NPs were the same as before lyophilization, confirming the long-term storage. Based on the results, as mentioned previously, the biological part of the study was conducted with Prot.1,2 (control) and Prot.1,2/RSP $(20 \% \mathrm{w} / \mathrm{w})$ NPs.

In vitro cell viability following $48 \mathrm{~h}$ treatment with Prot.1, Prot.2, and corresponding RSP-loaded NPs on cell proliferation of J774A.1 and Neuro- $2 \alpha$ cells was determined by XTT, as illustrated in Fig. 4. Prot.1/RSP and Prot.2/RSP demonstrates no cytotoxicity on either cell line. These results indicate that RSP-loaded NPs do not affect the metabolic viability of the cells and are suitable candidates as drug carriers for antipsychotic drugs.

The NIR dye Cy7 was conjugated to the surface of the NPs in order to access cellular uptake by Neuro- $2 \alpha$ cells. Figure 5A exhibits the intracellular fluorescence of Prot.1,2 and Prot.1,2/RSP obtained by FACS. The RSPencapsulating proteinoid NPs exhibited a slightly higher fluorescence compared to their hollow counterparts.

Cells were also observed by a confocal fluorescent microscope, as shown in Fig. 5b. The images demonstrate that following $4 \mathrm{~h}$ of incubation, both Prot. 1 and Prot. 2 penetrate the cell and reach the cytoplasm. These results support the hypothesis that proteinoid NPs resemble proteins and can therefore be transported across the cell membrane in a similar manner.

In order to learn about the body distribution and blood half-life (Fig. 6), blood was drawn at different time points, and $4 \mathrm{~h}$ post injection the mice were sacrificed and
Table 4 Composition of the different proteinoids

\begin{tabular}{llll}
\hline Proteinoid & L-Amino acid content & Weight ratio & PLLA (w\%) \\
\hline Prot.1 & Lys, Phe, His & $1: 1: 1$ & 10 \\
Prot.2 & Glu, Phe, His & $1: 1: 1$ & 10 \\
Prot.3 & Lys, Phe, His & $1: 1: 1$ & - \\
Prot.4 & Glu, Phe, His & $1: 1: 1$ & - \\
\hline
\end{tabular}

The total weight of the monomers content is $5 \mathrm{~g}$

organs were harvested. The half-life of both proteinoid NPs was calculated to be $20 \mathrm{~min}$. In both cases, fluorescence was seen in internal organs related to the gastrointestinal and urinal systems, which probably indicates that the NPs are eliminated from the body through the liver-intestine-colon and kidney-urine routes (Fig. 6b). Prot. 2 was not seen in the colon $4 \mathrm{~h}$ post injection, possibly due to fast clearance of Prot.2 from the colon or the urinary tract. Interestingly, a specific fluorescent signal was detected in the brain, indicating that both proteinoids can cross the BBB.

When comparing the fluorescence in the brain to the fluorescence in all tested organs, $\sim 1 \%$ of the total signal was found in the brain after $4 \mathrm{~h}$. Figure $6 \mathrm{c}$ exhibits a notable difference between the fluorescent intensity of Prot.1 NPs compared to Prot.2 NPs in the brain tissue $4 \mathrm{~h}$ post injection. This presumably stems from the difference in number of conjugated Cy7 molecules on Prot.1 NPs prior to injection, which is also demonstrated at time zero in Fig. 6a. The results indicate that both Prot.1 and Prot.2 can potentially be used as drug carriers to transport therapeutics across the BBB and target the brain.

The results of the current study support our hypothesis that RSP loaded within a proteinoid NP delivery system can stabilize and increase the bioavailability of the drug and thereby enhance its activity. Our data clearly demonstrate that RSP encapsulated in proteinoid NPs can cross the BBB and act at its receptor targets. Moreover, the effects of RSP-loaded proteinoid NPs appear to be stronger than those of free RSP, suggesting higher efficacy at the receptor targets. A possible explanation for this difference is the NPs' ability to penetrate into relevant brain areas and release the encapsulated RSP in the specific location. Furthermore, the encapsulated RSP is protected from decomposition by metabolic processes due to its entrapment within the proteinoid.

Nevertheless, several points should be addressed regarding these results. One interesting point is that RSP reduced activity both before and after amphetamine administration (Fig. 7). With the amphetamine-induced hyperactivity model, we expected that a tested compound at the appropriate dose would ameliorate amphetamineinduced activity but not baseline activity levels $[67,68]$. 
A<smiles>NC(CCC(=O)O)C(=O)O</smiles>

B<smiles>NCCCC(N)(O)O</smiles><smiles>O=C1CCC(C(=O)O)N1</smiles><smiles>[R]C(N)C(=O)O</smiles>

$\mathrm{R}=$ Amino acid side chain



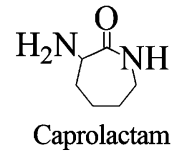

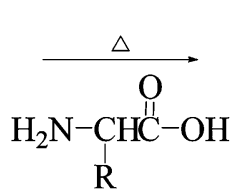

$\mathrm{R}$

$\mathrm{R}=$ Amino acid side chain

Fig. 8 General scheme of proteinoid synthesis. Step-growth polymerization of amino acids through pyroglutamic acid (a) or caprolactam (b) to obtain acidic and basic proteinoids, respectively

However, it has long been noted that antipsychotic drugs induce sedation and therefore reduce activity levels in both humans [69] and animal models [70]. The induction of sedation in untreated versus amphetamine-treated mice is therefore dependent on dosage, whereby a higher dose can induce sedation and consequently reduce activity regardless of amphetamine treatment. It is possible that a detailed dose-response study can identify the dose of RSP in proteinoid NPs that will ameliorate amphetamine-induced hyperactivity with no effects on baseline locomotion. However, that was not the aim of this study in which the in vivo behavioral testing was designed merely as a proof of concept study for behavioral effects of RSP administered in a proteinoid NP drug delivery system compared with free RSP.

Beyond measures of activity, the results show that the time spent in the center of the open field arena was reduced by amphetamine but significantly increased by RSP in proteinoid NPs. Time in the center of an open field can be interpreted in the context of anxiety where procedures that increase anxiety result in lower time spent in the center, while procedures that reduce anxiety (such as administration of anxiolytics) increase time spent in the center [71]. The anxiogenic effects of amphetamine are well known [72] and therefore its effects to reduce center time in the current study are not surprising. However, the effect of encapsulated RSP to drastically increase time in the center of the open field may be interpreted in the context of anxiety but is more likely to be the result of the drastically lower locomotor activity, with possible freezing of some of the animals in the center of the open field. As animals in the test were placed in the center of the arena, it is possible that the long time in the center results from freezing and is unrelated to anxiety. It is indeed well known that high doses of antipsychotic drugs, including RSP, can induce catalepsy [73]. This is reinforced by the current study in which some of the animals spent over $1700 \mathrm{~s}$ (of an $1800 \mathrm{~s}$ session, before or after amphetamine treatment) in the center area with only minimal locomotor activity. The freezing response in animals prior to amphetamine treatment suggests caution in future translational studies as it is possible that relatively low doses will be enough to induce catalepsy. Yet, careful dose/ response studies in humans should be sufficient to identify appropriate doses with stronger antipsychotic effects but without induction of catalepsy, as is the case for most antipsychotic treatments.

However, despite the limitations, it is clear that RSP encapsulated within proteinoid NPs and administered via peripheral injection reached the brain and induced strong and significant behavioral effects, as expected from a high dose of an antipsychotic drug. Therefore, it can be assumed that the encapsulated RSP is more effective than free risperidone.

\section{Conclusions}

In this study, a new drug delivery system was investigated for the antipsychotic drug RSP. For this purpose, basic/ acidic proteinoids were synthesized from L-Lys/Glu, LPhe, L-His and PLLA via a step-growth polymerization process, yielding polymers with relatively high molecular weight and narrow size distribution. Hollow and RSPloaded proteinoid NPs were prepared by self-assembly process, followed by PEGylation, and characterized for

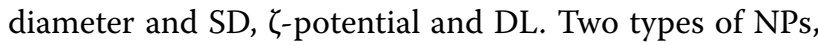
Prot.1 and Prot.2, were chosen for further investigation based on diameter and stability and examined with various drug concentrations by HPLC, yielding optimal DL of $20 \% \mathrm{w} / \mathrm{w}$.

The hollow and optimal RSP-loaded NPs were characterized by DLS, cryo-TEM and HR-SEM. The measurements confirmed that RSP was successfully encapsulated, 
resulting in nano-sized particles with diameters of $97 \pm 4$ and $86 \pm 3 \mathrm{~nm}$ for Prot.1 and Prot.2, respectively. After long-term storage by freeze-drying in the presence of $1 \%$ $\mathrm{w} / \mathrm{v}$ trehalose, particles were redispersed successfully to the original size.

In vitro studies of cytotoxicity and NP cell uptake by XTT, FACS and fluorescence microscopy demonstrated that both hollow and encapsulating NPs are non-toxic, penetrate the cell membrane, and reach the cytoplasm. In vivo biodistribution studies showed that Prot.1 and Prot. 2 were distributed to all examined organs, including the brain after passing the BBB.

The antipsychotic effect of the RSP-loaded NPs was examined by behavioral testing using the amphetamineinduced hyperactivity screening model in mice. The results supported the hypothesis that the effect of RSP increases when entrapped within NPs. This may probably be explained by increased drug solubility and stability of the encapsulated RSP, leading to increased transportation across the $\mathrm{BBB}$ and better penetration to the specific area in the brain compared to the free drug. Hence, both Prot.1/RSP and Prot.2/RSP demonstrated excellent in vivo activity in reducing the psychotic behavior compared to free RSP, and can therefore potentially be used as an effective drug delivery system for RSP and other antipsychotic drugs. Future research envisages assessment of a wide range of behavioral tests and side effects of RSP-loaded proteinoid NPs at different concentrations. Ongoing studies of RSP-loaded proteinoids focus on optimal dosage and investigation of side effects for antipsychotic effect as well as for treatment of hyperactivity disorders.

\section{Methods}

\section{Materials}

The following analytical-grade chemicals were purchased from Sigma (Israel) in $\geq 98 \%$ purity and used without further purification: L-glutamic acid (Glu), L-lysine (Lys), L-phenylalanine (Phe), L-histidine (His), sodium hydroxide $(\mathrm{NaOH})$, super-pure HPLC water, acetonitrile, trifluoroacetic acid (TFA), sodium chloride $(\mathrm{NaCl})$, dimethyl sulfoxide (DMSO), trehalose, and Cyanine7NHS (Cy7-NHS). Poly(L-lactic acid) (PLLA) MW 2 kDa was purchased from Polysciences (Warrington, PA, USA), methoxy polyethylene glycol succinimidyl succinimide (M-PEG-NHS, Mw $5 \mathrm{kDa}$ ) was obtained from JenKem Technology (Plano, TX, USA), and Risperidone was acquired from Teva Pharmaceutical Industries Ltd. (Kfar Saba, Israel). Water was purified by passing deionized water through an Elgastat Spectrum reverse osmosis system from Elga Ltd. (High Wycombe, UK). Dialysis membranes (1000 Da), Dulbecco's modification of Eagle's medium (DMEM), phosphate buffered saline (PBS), glutamine, penicillin/streptomycin, and XTT-based cell proliferation kit were purchased from Biological Industries (Bet Haemek, Israel). J774A.1 murine macrophages and Neuro- $2 \alpha$ neuroblast cell lines were purchased from ATCC (Manassas, VA, USA). BALB/c and ICR (CD-1) mice were purchased from Harlan Biotech (Rehovot, Israel).

\section{Synthesis of proteinoids by thermal step-growth polymerization mechanism}

Proteinoid synthesis was carried out as described in Table 4, in absence or presence of $2 \mathrm{kDa}$ PLLA (10 w\%) [63]. In brief, under $\mathrm{N}_{2}$ atmosphere, $5 \mathrm{~g}$ of Lys or Glu, Phe and His at 1:1:1 weight ratio was heated at either 140 or $180{ }^{\circ} \mathrm{C}$, respectively. The mixture was stirred by a mechanical stirrer at $150 \mathrm{rpm}$ for $45 \mathrm{~min}$. The product was a highly viscous amber-brownish paste, which hardened upon cooling to room temperature. The residue was extracted by $30 \mathrm{~mL}$ of super-purified water, followed by lyophilization to yield dried proteinoid polymer powder.

\section{Proteinoid characterization}

The molecular weights and polydispersity indices of the dried crude proteinoids were determined at $25{ }^{\circ} \mathrm{C}$ using GPC consisting of a Waters Spectra Series P100 isocratic HPLC pump with an ERMA ERC-7510 refractive index detector and a Rheodyne (Coatati, CA) injection valve with a $20 \mu \mathrm{L}$ loop (Waters, MA). The samples were eluted with super-pure HPLC water through a linear BioSep SEC-s3000 column (Phenomenex) at a flow rate of $1 \mathrm{~mL} /$ min. The molecular weights of the proteinoids were determined relative to PEG standards (Polymer Standards Service-USA, Silver Spring, MD) with a Mw range of 100-450,000 Da, human serum albumin (HSA, $67 \mathrm{kDa}$ ) and bovine plasma fibrinogen $(340 \mathrm{kDa})$ using Clarity chromatography software version 2.7.3.498 (DataApex, Prague, Czech Republic).

The optical activities of the proteinoids were determined with a PE 343 polarimeter (PerkinElmer). All measurements were performed in water at $589 \mathrm{~nm}$ and $25^{\circ} \mathrm{C}$.

\section{Preparation of hollow and RSP-loaded proteinoid NPs}

Proteinoid NPs were prepared via self-assembly mechanism. Briefly, $100 \mathrm{mg}$ of the dried proteinoid were added to $10 \mathrm{~mL} \mathrm{NaCl} 10 \mu \mathrm{M}$ aqueous solution. The mixture was heated to $80{ }^{\circ} \mathrm{C}$ and stirred at $250 \mathrm{rpm}$ for $30 \mathrm{~min}$ until the crude proteinoid dissolved completely. Hollow proteinoid particles were formed as the mixture was left to cool to room temperature. RSP-loaded proteinoid particles were obtained in a similar procedure by the addition of RSP to the heated proteinoid solution. Due to the poor solubility of RSP in water, an appropriate concentration 
of RSP powder $(0,10,20,30,40$ and 50 weight\% relative to the proteinoid) was first dissolved in DMSO $(0.2 \mathrm{~mL})$ and then heated to $80{ }^{\circ} \mathrm{C}$. After both solutions reached $80{ }^{\circ} \mathrm{C}$, RSP solution was added to the proteinoid mixture prior to the particle formation to achieve RSP-loaded proteinoid NP dispersion. Following preparation, the NPs were extensively dialyzed using a cellulose dialysis membrane with a molecular weight cut-off (MWCO) of 1000 Da against distilled water to remove the DMSO. The particle dispersion was filtered through a $3 \mu \mathrm{m}$ glass microfiber membrane syringe filter (VWR EU, England) to remove excess RSP.

\section{PEGylation of hollow and RSP-loaded proteinoid NPs}

PEGylated RSP-loaded proteinoid NPs (Prot./RSP) were prepared with M-PEG-NHS, Mw $5000 \mathrm{Da}$, which was reacted with the primary amine groups on the surface of the proteinoid NPs. Briefly, $100 \mu \mathrm{L}$ of M-PEG-NHS dissolved in PBS (50 mg/mL) were added to RSP-loaded NPs $(10 \mathrm{mg} / \mathrm{mL})$ dispersed in sodium bicarbonate $(2 \mathrm{mM}$, $\mathrm{pH}=8)$ and the mixture was stirred at $150 \mathrm{rpm}$ for $2 \mathrm{~h}$ at room temperature. The obtained PEGylated RSP-loaded NPs dispersions were then dialyzed through a cellulose membrane (1000 Da MWCO) against super-purified water to remove excess undesired materials. Similarly, PEGylation process was performed for the hollow proteinoid NPs.

\section{Preparation of NIR fluorescent PEGylated hollow and RSP-loaded proteinoid NPs}

The NIR dye Cyanine7 NHS ester (Cy7-NHS) was conjugated to residual amine groups on the surface of the PEGylated hollow and RSP-loaded proteinoid NPs. Briefly, after coupling of the M-PEG-NHS, before dialysis, as described above, $50 \mu \mathrm{L}$ of Cy7-NHS solution in DMSO $(10 \mathrm{mg} / \mathrm{mL})$ were added to the aqueous NP dispersion and stirred at $150 \mathrm{rpm}$ for additional $2 \mathrm{~h}$ at room temperature. The NIR fluorescent conjugated NP dispersion was then extensively dialyzed through a cellulose membrane (1000 Da MWCO) against distilled water to remove the DMSO and excess Cy7.

\section{Proteinoid NP characterization \\ Diameter and size distribution}

The hydrodynamic diameter and size distribution of the aqueous NP dispersions were measured at room temperature with a particle DLS analyzer model Nanophox (Sympatec $\mathrm{GmbH}$, Germany). In addition, the diameter and size distribution of the NPs were measured with a cryogenic transmission electron microscope (cryo-TEM). Briefly, a small droplet of NP aqueous dispersion was placed on a perforated lacey carbon film supported on a TEM copper grid. The drop was blotted with a piece of filter paper, resulting in formation of thin films of
100-300 $\mathrm{nm}$. The specimen was subsequently plunged into a reservoir of liquid ethane cooled by liquid nitrogen to ensure its vitrification (rapid freezing) and to prevent ice crystal formation. The vitrified specimen was transferred under liquid nitrogen and mounted on a cryogenic sample holder cooled to $-170{ }^{\circ} \mathrm{C}$. All samples were observed under low-dose conditions. Vitrified samples were examined using a FEI T12 G2 Cryo-TEM operating at $120 \mathrm{kV}$ and equipped with a Gatan 626 cryoholder system. The mean diameter was determined by measuring at least 200 particles using the AnalySIS Auto image analysis software version 3.2 (Soft Imaging System GmbH, Germany).

Dried particle diameter and size distribution were measured with a scanning electron microscope (SEM, JEOL, JSM-840 Model, Japan). A drop of dilute particle dispersion in distilled water was spread on a glass surface and dried at room temperature. The dried sample was coated with carbon in vacuum before viewing under SEM. The average particle diameter and distribution were determined by measuring the diameter of more than 200 particles with the AnalySIS image analysis software.

\section{$\zeta$-Potential}

The proteinoid NP surface potentials were measured in aqueous dispersion at $\mathrm{pH}=7.4$ at a concentration of $10 \mathrm{mg} / \mathrm{mL}$ using a Zetasizer $3000 \mathrm{HSa}$ model $\zeta$-potential analyzer (Malvern Instruments Company, England).

\section{HPLC}

HPLC analysis of the RSP drug loading (DL) was carried out by a Spectra System HPLC equipped with a UV/vis detector (Thermo Scientific, USA) and a reverse phase C18 column (75 $\mathrm{mm} \times 4.6 \mathrm{~mm}$, Phenomenex, USA). The mobile phase was water and acetonitrile, both containing $0.1 \%$ aqueous TFA solution, at a flow rate of $1 \mathrm{~mL} / \mathrm{min}$ at $25^{\circ} \mathrm{C}$ wavelength was set at $285 \mathrm{~nm}$ [74]. Calibration standard solutions were prepared and used by diluting an appropriate volume of stock standard solution in ethanol, yielding concentrations of RSP in the range of 60-200 $\mu \mathrm{M}$. RSP-loaded NPs were diluted by ethanol and sonicated in an ice-water bath for 20 min prior to injection. The sonication and the added ethanol cause the proteinoid NPs to disassemble and elute the RSP. The injection volume was set to $10 \mu \mathrm{L}$ for all standard samples in the range of 60-200 $\mu$ M RSP. The weight of drug in each sample was calculated using the calibration curve.

\section{Leakage of RSP}

A dialysis technique was applied to study RSP leakage from the Prot./RSP NPs [7]. Briefly, RSP (20\% w/w) loaded proteinoid aqueous dispersions $(10 \mathrm{mg} / \mathrm{mL})$ were 
kept in the refrigerator at $4{ }^{\circ} \mathrm{C}$ for 3 months followed by extensive dialysis against water. The diameter and size distribution of the RSP-loaded proteinoid NP aqueous dispersions and their RSP content were measured by DLS and HPLC, respectively.

\section{Proteinoid NPs long term storage stability}

Long-term storage of the Prot./RSP NPs was investigated by freeze-drying methodology. Briefly, $10 \mathrm{mg}$ of trehalose were added to $1 \mathrm{~mL}$ of NP aqueous dispersion $(10 \mathrm{mg} /$ $\mathrm{mL}$ ), followed by lyophilization to dryness and storage at $4{ }^{\circ} \mathrm{C}$. The NP powder was redispersed in water to the original concentration (in $1 \mathrm{~mL}$ ) and the diameter and size distribution were determined by DLS.

\section{Cell viability analysis for proteinoid NPs}

In vitro toxicity of PEGylated hollow and RSP-loaded NPs was tested using the XTT (2,3-bis-(2-methoxy4-nitro-5-sulfophenyl)-2H-tetrazolium-5-carboxanilide-salt) assay in two murine cell lines: J774A.1 macrophage and Neuro- $2 \alpha$ neuroblast cells. The cells were seeded in a 96-well plate at a density of $1 \times 10^{4}$ cells/well in $100 \mu \mathrm{L}$ culture medium and grown in a humidified $5 \% \mathrm{CO}_{2}$ atmosphere at $37{ }^{\circ} \mathrm{C}$. The PEGylated hollow and RSP-loaded NPs were freshly dispersed in water containing 1\% DMSO aqueous solution, and NPs dispersions were added to $95 \%$ confluent cell culture in culture medium.

Cells were treated with PEGylated hollow and RSPloaded NPs at a concentration of $0.5 \mathrm{mg} / \mathrm{mL}$ with RSP concentration of $10 \mu \mathrm{g} / \mathrm{mL}$. Cells treated with an equivalent amount of free RSP and untreated cells served as control groups. The cell cultures were further incubated at $37{ }^{\circ} \mathrm{C}$ in a humidified $5 \% \mathrm{CO}_{2}$ incubator and examined for cellular viability after $48 \mathrm{~h}$. The cell viability was calculated as shown in the manufacturer's protocol of the XTT kit, and the experiment was repeated twice. All samples were tested in six-fold and analyzed by UV spectrophotometer at $492 \mathrm{~nm}$ with reference wavelength of $620 \mathrm{~nm}$. The assay assesses the metabolic rate of the mitochondria in the cells following NP exposure.

\section{Proteinoid NP cell uptake}

The ability of proteinoid NPs to penetrate the cell membrane was studied using flow cytometry. FACS of Cy7conjugated NPs within the cells was evaluated by a FACS Aria III (BD) cell sorter. Cells were treated with Cy7conjugated PEGylated hollow and RSP-loaded Prot.1 and Prot. 2 NPs $(0.5 \mathrm{mg} / \mathrm{mL})$ for $24 \mathrm{~h}$ at $37{ }^{\circ} \mathrm{C}$ prior to analysis. After incubation, the cells were washed with fresh medium and stained with Hoechst 33342 (nucleus staining, $1 \mu \mathrm{g} / \mathrm{mL}$ ). In order to maximize cell viability and minimize mechanical perturbations, the flow rate was set to 1.1 (minimum). For Cy7 analysis, a $633 \mathrm{~nm}$ excitation laser was used with a filter. The data was processed by FlowJo v7.6.4. Live cell imaging was performed on an Olympus FV-1000 confocal microscope.

For the Cy7-conjugated particles, 50,000 Neuro- $2 \alpha$ cells were grown on $15 \mathrm{~mm}$ glass coverslips pretreated with $0.1 \%$ gelatin for $48 \mathrm{~h}$ Cells and treated with Cy7conjugated PEGylated hollow NPs and Prot. 1,2/RSP NPs $(0.1 \mathrm{mg} / \mathrm{mL})$ for $24 \mathrm{~h}$ at $37^{\circ} \mathrm{C}$. After incubation, cells were washed with PBS, fixed by cold $\left(-20{ }^{\circ} \mathrm{C}\right)$ methanol for 20 min and stained with DAPI (nucleus staining, Fluoroshield, Sigma). Cell imaging was performed on the Olympus confocal microscope.

\section{In vivo biodistribution studies}

Male BALB/C mice $(\mathrm{n}=12$, Harlan Laboratories, Israel) were utilized under a protocol approved by the Institutional Animal Care and Use Committee at Bar-Ilan University. The biodistribution of the Cy7 fluorescent NPs was studied in normal 8-week-old mice weighing 25-30 g at the time of experiment.

For this purpose, $100 \mu \mathrm{L}$ of the Cy7-conjugated PEGylated hollow proteinoid and Prot./RSP NPs and PBS dispersion $(0.01 \mathrm{mg} / \mathrm{kg}$ body weight) were administered to the mice via IV injection to the tail vein at a concentration of $0.3 \mathrm{mg} / \mathrm{kg}$. Blood samples were taken immediately after NP injection at $0,30 \mathrm{~min}, 1 \mathrm{~h}$ and $4 \mathrm{~h}$, mice were euthanized by $\mathrm{CO}_{2} 4 \mathrm{~h}$ post injection, and organs were harvested for imaging (brain, colon, heart, lungs, liver, spleen, kidneys, duodenum and blood). Each group consisted of five mice, and two nontreated mice served as negative control.

Fluorescence images were acquired using a Maestro II in vivo fluorescence imaging system (Cambridge Research \& Instrumentation, Inc., Woburn, MA) The system is equipped with a fiber-delivered $300 \mathrm{~W}$ xenon excitation lamp, and images can be acquired from $\lambda=500-950 \mathrm{~nm}$ by a 1.3 megapixel CCD camera (Sony ICX285 CCD chip). A deep red excitation/emission filter set was used for our experiments $(\lambda$ ex: $700-770 \mathrm{~nm}$, $\lambda \mathrm{em}>780 \mathrm{~nm}$ ). The liquid crystal tunable filter was programmed to acquire image cubes from $\lambda=780$ to $840 \mathrm{~nm}$ with an increment of $10 \mathrm{~nm}$ per image. The camera was set to exposure times of 20,000 ms (brain), $400 \mathrm{~ms}$ (colon), $2000 \mathrm{~ms}$ (heart), $1000 \mathrm{~ms}$ (lungs), $3000 \mathrm{~ms}$ (liver), 20,000 ms (spleen), $3000 \mathrm{~ms}$ (kidneys), $3000 \mathrm{~ms}$ (duodenum), and $5000 \mathrm{~ms}$ (blood). Fluorescence intensity measurements were performed using ImageJ NIH (National Institutes of Health) software version $1.48 \mathrm{v}$. 


\section{In vivo psychopharmacological studies}

An amphetamine-induced hyperactivity test was used to examine the possible effects of PEGylated hollow proteinoid and Prot.1,2/RSP NP dispersions at the behavioral level. Subjects were male ICR $\left(\mathrm{CD}-1^{\circledR}\right)$ outbred 8-week-old mice $(n=48)$ weighing $25-30 \mathrm{~g}$ at the time of experiment. Mice were treated with either saline (control), free RSP, or NP dispersion. Specifically, $100 \mu \mathrm{L}$ of either NP dispersion at a concentration of $2 \mathrm{mg} / \mathrm{mL}$ or a solution of $10 \mathrm{mg}$ RSP dissolved previously with $1 \%(\mathrm{v} / \mathrm{v})$ DMSO in $5 \mathrm{~mL}$ super-purified water, giving a final concentration of $2 \mathrm{mg} / \mathrm{mL}$, and saline were injected IV via the tail to the mice, immediately before the start of the behavioral test according to the $t_{1 / 2}$ of the NP dispersion in the blood. Following treatment, each mouse was placed in the center of a $40 \times 40 \mathrm{~cm}$ open field arena with $30 \mathrm{~cm}$ high walls where activity was monitored using the ANY-maze automated video tracking system (Stoelting, IL, United States) for a $60 \mathrm{~min}$ session. Thirty min after the start of the session, mice were briefly taken out of the arena, injected IP with amphetamine dissolved in saline at a $5.0 \mathrm{mg} / \mathrm{kg}$ dose and $10 \mathrm{~mL} / \mathrm{kg}$ volume, and immediately placed back in the arena for additional $30 \mathrm{~min}$. This is a within subject design for the effects of amphetamine and across subject design for the effects of RSP. The experiment was repeated twice.

\section{Statistical analysis}

Data for the behavioral experiment were analyzed using Statistica 13.0 (Dell, Texas) software. Analysis included a mixed ANOVA with RSP treatment as a main factor and amphetamine administration as a repeated measures factor (pre and post amphetamine injection). Significant effects were followed by Scheffe post hoc tests. Effect sizes (Cohen's d) were calculated using an online calculator (https://www.uccs.edu/lbecker/). One mouse from group Prot.1/RSP was excluded from the statistical analysis as an outlier (more than two standard deviations away from the group mean).

\section{Ethical approval}

All experiments complied with the ARRIVE guidelines (Animal Research: Reporting of In Vivo Experiments) and were carried out in accordance with the National Institute of Health and the Israeli Ministry of Health Guide for the Care and Use of Laboratory Animals (NIH Publications No. 80-23). All procedures were approved by the Bar-Ilan University Institutional Animal Care and Use Committee (IACUC).
Abbreviations

ANOVA: Analysis of variance; Cryo-TEM: Cryogenic transmission electron microscopy; DL: Drug loading; DLS: Dynamic light scattering; GPC: Gel permeation chromatography; HPLC: High performance liquid chromatography; IP: Intraperitoneal; IV: Intravenous; M-PEG-NHS: Methoxy polyethylene glycol succinimidyl succinimide; NPs: Nanoparticles; NIR: Near infrared; PDI: Polydispersity index; PLLA: Poly(L-lactic) acid; Prot./RSP: Risperidone-loaded proteinoid; RSP: Risperidone; SEM: Scanning electron microscope; SD: Size distribution; XTT: 2,3-Bis-(2-methoxy-4-nitro-5-sulfophenyl)-2H-tetrazolium-5-carboxanilide.

\section{Acknowledgements}

The authors thank Dr. Alexander Varvak for assistance with HPLC analysis, Dr. Ronen Yehuda for assistance with fluorescent images, Rivka Sabag for assistance with the animal experiments, and Dr. Yuval Elias for scientific editing of the manuscript.

\section{Authors' contributions}

LL designed, prepared, characterized and investigated the different NPs, performed and analyzed the in vitro and in vivo experiments, and drafted the manuscript; IG carried out and analyzed the in vitro and in vivo experiments; RGS characterized the NPS and revised the manuscript; EO, behavioral science investigator; $\mathrm{HE}$, behavioral science investigator; SM, principal investigator. All authors read and approved the final manuscript.

\section{Funding}

This research received no external funding.

\section{Availability of data and material}

All data generated or analyzed during this study are included in this published article.

\section{Ethical approval}

All experiments in the research were conducted under a protocol approved by the Institutional Animal Care and Use Committee at Bar-Ilan University. Protocol reference numbers 16-09-2019.

\section{Consent for publication}

Not applicable.

\section{Competing interests}

The authors declare that they have no competing interests.

\section{Author details}

1 Department of Chemistry, The Institute of Nanotechnology and Advanced Materials, Bar-llan University, 5290002 Ramat Gan, Israel. ${ }^{2}$ The Mina and Everard Goodman, Faculty of Life Sciences, Bar-llan University, 5290002 Ramat Gan, Israel. ${ }^{3}$ The School of Behavioral Sciences, Tel Aviv-Yaffo Academic College, 6818211 Tel Aviv, Israel.

Received: 19 June 2020 Accepted: 9 October 2020

Published online: 21 October 2020

References

1. Stein DJ, Phillips KA, Bolton D, Fulford KWM, Sadler JZ, Kendler KS. What is a mental/psychiatric disorder? From DSM-IV to DSM-V. Psychol Med. 2010;40:1759-65.

2. Reichborn-Kjennerud T. The genetic epidemiology of personality disorders. Dialogues Clin Neurosci. 2010;12:103-14.

3. Saia-Cereda VM, Cassoli JS, Martins-de-Souza D, Nascimento JM. Psychiatric disorders biochemical pathways unraveled by human brain proteomics. Eur Arch Psychiatry Clin Neurosci. 2017;267:3-17.

4. Tyrer P. The importance of nidotherapy and environmental change in the management of people with complex mental disorders. Int J Environ Res Public Health. 2018;15:972.

5. Farina $B$, Liotti $M$, Imperatori $C$. The role of attachment trauma and disintegrative pathogenic processes in the traumatic-dissociative dimension. Front Psychol. 2019;10:933.

6. Conway KP, Swendsen J, Husky MM, He JP, Merikangas KR. Association of lifetime mental disorders and subsequent alcohol and illicit drug use: 
results from the national comorbidity survey-adolescent supplement. J Am Acad Child Adolesc Psychiatry. 2016;55:280-8.

7. Das S, Ng WK, Kanaujia P, Kim S, Tan RBH. Formulation design, preparation and physicochemical characterizations of solid lipid nanoparticles containing a hydrophobic drug: effects of process variables. Colloids Surf B Biointerfaces. 2011;88:483-9.

8. Sajatovic M, Subramoniam M, Fuller MA. Risperidone in the treatment of bipolar mania. Neuropsychiatr Dis Treat. 2006;2:127-38.

9. Danel C, Azaroual N, Brunel A, Lannoy D, Vermeersch G, Odou P, et al. Study of the complexation of risperidone and 9-hydroxyrisperidone with cyclodextrin hosts using affinity capillary electrophoresis and $1 \mathrm{H} \mathrm{NMR}$ spectroscopy. J Chromatogr A. 2008;1215:185-93.

10. Hannah-Poquette C, Anderson GW, Flaisher-Grinberg S, Wang J, Meinerding TM, Einat H. Modeling mania: further validation for Black Swiss mice as model animals. Behav Brain Res. 2011;223:222-6.

11. Cohen LJ. Risperidone. Pharmacother J Hum Pharmacol Drug Ther. 1994;14:253-65.

12. Rogóż Z. Effect of co-treatment with mirtazapine and risperidone in animal models of the positive symptoms of schizophrenia in mice. Pharmacol Reports. 2012;64:1567-72.

13. Sun F, Su Z, Sui C, Zhang C, Yuan L, Meng Q, et al. Studies on the acute toxicity, pharmacokinetics and pharmacodynamics of paliperidone derivatives - Comparison to paliperidone and risperidone in mice and rats. Basic Clin Pharmacol Toxicol. 2010;107:656-62.

14. El-Sherif ZA, El-Zeany B, El-Houssini OM. High performance liquid chromatographic and thin layer densitometric methods for the determination of risperidone in the presence of its degradation products in bulk powder and in tablets. J Pharm Biomed Anal. 2005;36:975-81.

15. Uçok A, Gaebel W. Side effects of atypical antipsychotics: a brief overview. World Psychiatry. 2008;7:58-62.

16. Lu Y, Chen SC. Micro and nano-fabrication of biodegradable polymers for drug delivery. Adv Drug Deliv Rev. 2004;56:1621-33.

17. Leone-Bay A, McInnes C, Wang NF, DeMorin F, Achan D, Lercara C, et al. Microsphere formation in a series of derivatized a-amino acids: properties, molecular modeling, and oral delivery of salmon calcitonin. J Med Chem. 1995;38:4257-62.

18. Leone-bay A, Santiago N. N-acylated alpha-amino acids as novel oral delivery agents for proteins. J Med Chem. 1995;38:4263-9.

19. Hans ML, Lowman AM. Biodegradable nanoparticles for drug delivery and targeting. Curr Opin Solid State Mater Sci. 2002;6:319-27.

20. Karlsson J, Vaughan HJ, Green JJ. Biodegradable polymeric nanoparticles for therapeutic cancer treatments. Annu Rev Chem Biomol Eng. 2018;9:105-27.

21. George A, Shah PA, Shrivastav PS. Natural biodegradable polymers based nano-formulations for drug delivery: a review. Int J Pharm. 2019;561:244-64.

22. Naskar S, Kuotsu K, Sharma S. Chitosan-based nanoparticles as drug delivery systems: a review on two decades of research. J Drug Target. 2019;27:379-93.

23. Saraiva C, Praça C, Ferreira R, Santos T, Ferreira L, Bernardino L. Nanoparticle-mediated brain drug delivery: overcoming blood-brain barrier to treat neurodegenerative diseases. J Control Release. 2016;235:34-47.

24. Pardridge WM. Blood-brain barrier and delivery of protein and gene therapeutics to brain. Front Aging Neurosci. 2020;11:373.

25. Fox SW. How did life begin? Science. 1960;132:200-8.

26. Fox SW. The proteinoid theory of the origin of life and competing ideas. Am Biol Teach. 1974;36:161-81.

27. Fox SW. Thermal synthesis of amino acids and the origin of life. Geochim Cosmochim Acta. 1995;59:1213-4.

28. Fox SW, Jungck JR, Nakashima T. From proteinoid microsphere to contemporary cell: formation of internucleotide and peptide bonds by proteinoid particles. Orig Life. 1974;5:227-37.

29. Fox SW, Mccauley RJ, Fukushima T, Windsor CR, Montgome PO. Selective action in boundaries of particles of thermal proteinoid. Fed Proc. 1967;26:749.

30. Fox SW, Nakashima T, Przybylski A, Syren RM. The updated experimental proteinoid model. Int J Quantum Chem. 1982;22:195-204.

31. Kumar AA, Karthick K, Arumugam KP. Properties of biodegradable polymers and degradation for sustainable development. Int J Chem Eng Appl. 2011;2:164-7.
32. Kile S, Kolitz-Domb M, Corem- Salkmon E, Margel S. Engineered doxorubicin delivery system using proteinoid-poly (L-lactic acid) polymeric manoparticles of narrow size distribution and high molecular weight for cancer treatment. Int J Nanotechnol Nanomed. 2017;2:1.

33. Kolitz-Domb M, Margel S. Recent advances of novel proteinoids and proteinoid nanoparticles and their applications in biomedicine and industrial uses. Isr J Chem. 2018:58:1277-85.

34. Shikanov A, Kumar N, Domb AJ. Biodegradable polymers: an update. Isr J Chem. 2005:45:393-9.

35. Syren RM, Sanjur A, Fox SW. Proteinoid microspheres more stable in hot than in cold water. BioSystems. 1985;17:275-80.

36. Madhan Kumar AB, Panduranga Rao K. Preparation and characterization of $\mathrm{pH}$-sensitive proteinoid microspheres for the oral delivery of methotrexate. Biomaterials. 1998;19:725-32.

37. Urry DW, Channe Gowda D, Peng SQ, Parker TM. Non-linear hydrophobicinduced pKa shifts: implications for efficiency of conversion to chemical energy. Chem Phys Lett. 1995;239:67-74.

38. Quirk S. Enhanced catalytic activity from proteinoid microspheres. J Biomed Mater Res, Part A. 2013;101A:1133-43.

39. Kolitz-Domb M, Corem-Salkmon E, Grinberg I, Margel S. Synthesis and characterization of bioactive conjugated near-infrared fluorescent proteinoid-poly (L-lactic acid) hollow nanoparticles for optical detection of colon cancer. Int J Nanomed. 2014;9:5041-53.

40. Kolitz-Domb M, Grinberg I, Corem-Salkmon E, Margel S. Engineering of near infrared fluorescent proteinoid-poly (L-lactic acid) particles for in vivo colon cancer detection. J Nanobiotechnol. 2014;12:30-43.

41. Kulkarni RK, Moore EG, Hegyeli AF, Leonard F. Biodegradable poly(lactic acid) polymers. J Biomed Mater Res. 1971;5:169-81.

42. Bala I, Hariharan S, Kumar MR. PLGA nanoparticles in drug delivery: the state of the art. Crit Rev Ther Drug Carrier Syst. 2004;21:387-422.

43. Danhier F, Ansorena E, Silva JM, Coco R, Le Breton A, Préat V. PLGAbased nanoparticles: an overview of biomedical applications. J Control Release. 2012;161:505-22.

44. Shi M, Lu J, Shoichet MS. Organic nanoscale drug carriers coupled with ligands for targeted drug delivery in cancer. J Mater Chem. 2009;19:5485-98.

45. Aggarwal P, Hall JB, McLeland CB, Dobrovolskaia MA, MCNeil SE. Nanoparticle interaction with plasma proteins as it relates to particle biodistribution, biocompatibility and therapeutic efficacy. Adv Drug Deliv Rev. 2009;61:428-37.

46. Gabizon A, Shmeeda H, Barenholz Y. Pharmacokinetics of PEGylated liposomal doxorubicin. Clin Pharmacokinet. 2003:42:419-36.

47. Tang BC, Dawson M, Lai SK, Wang YY, Suk JS, Yang M, et al. Biodegradable polymer nanoparticles that rapidly penetrate the human mucus barrier. Proc Natl Acad Sci. 2009;106:19268-73.

48. Nance EA, Woodworth GF, Sailor KA, Shih TY, Xu Q, Swaminathan G, et al. A dense poly(ethylene glycol) coating improves penetration of large polymeric nanoparticles within brain tissue. Sci Transl Med. 2012:4:149ra119.

49. Suk JS, Xu Q, Kim N, Hanes J, Ensign LM. PEGylation as a strategy for improving nanoparticle-based drug and gene delivery. Adv Drug Deliv Rev. 2016;99:28-51.

50. Einat $\mathrm{H}$, Shaldubina A, Bersudskey $\mathrm{Y}$, Belmaker RH. Prospects for the development of animal models for the study of bipolar disorder. In: Soares JC, Young A, editors. Bipolar disorders: basic mechanisms and therapeutic implications. New York: Taylor \& Francis; 2007.

51. Ene HM, Kara NZ, Einat H. The effects of the atypical antipsychotic asenapine in a strain-specific battery of tests for mania-like behaviors. Behav Pharmacol. 2015;26:331-7.

52. Marston HM, Young JW, Martin FDC, Serpa KA, Moore CL, Wong EHF, et al. Asenapine effects in animal models of psychosis and cognitive function. Psychopharmacology. 2009;206:699-714.

53. Latt SA, Stetten G, Juergens LA, Willard HF, Scher CD. Recent developments in the detection of deoxyribonucleic acid synthesis by 33258 Hoechst fluorescence. J Histochem Cytochem. 1975;23:493-505.

54. Fox SW, Harada K. The thermal copolymerization of amino acids common to protein. J Am Chem Soc. 1960;82:3745-51.

55. Kohn J, Langer R. Polymerization reactions involving the side chains of alpha-L-amino acids. J Am Chem Soc. 1987;109:817-20. 
56. Harada K, Matsuyama M. Polycondensation of thermal precursors of amino acids and characterization of constituent amino acids. BioSystems. 1979;11:47-53.

57. Fox SW, Waehneldt TV. The thermal synthesis of neutral and basic proteinoids. Biochim Biophys Acta. 1968;160:246-9.

58. Heinrich R. The effect of time of heating on the thermal pof L-Lysine. Arch Biochem Biophys. 1969;130:441-8.

59. Harada K, Fox SW. The thermal condensation of glutamic acid and glycine to linear peptides. J Am Chem Soc. 1958;80:2694-7.

60. Kumar ABM, Jayakumar R, Rao KP. Synthesis and aggregational behavior of acidic proteinoid. J Polym Sci, Part A: Polym Chem. 1996;34:2915-24.

61. Jiang T, Zhang Z, Zhang Y, Lv H, Zhou J, Li C, et al. Dual-functional liposomes based on $\mathrm{pH}$-responsive cell-penetrating peptide and hyaluronic acid for tumor-targeted anticancer drug delivery. Biomaterials. 2012;33:9246-58.

62. Stille JK. Step-growth polymerization. J Chem Educ. 1981;58:862.

63. Kolitz-Domb M, Margel S. Engineering of novel proteinoids and PLLAproteinoid polymers of narrow size distribution and uniform nano/microhollow particles for biomedical applications. Adv Bioeng. 2015;51-77.

64. Slager J, Domb AJ. Biopolymer stereocomplexes. Adv Drug Deliv Rev. 2003;55:549-83.

65. Das S, Chaudhury A. Recent advances in lipid nanoparticle formulations with solid matrix for oral drug delivery. AAPS PharmSciTech. 2011;12:62-76

66. Freitas C, Müller RH. Effect of light and temperature on zeta potential and physical stability in solid lipid nanoparticle $\left(S L N^{\mathrm{TM}}\right)$ dispersions. Int J Pharm. 1998;168:221-9.

67. Arnt J. Differential effects of classical and newer antipsychotics on the hypermotility induced by two dose levels of d-amphetamine. Eur J Pharmacol. 1995;283:55-62.

68. Kask A, Harro J. Inhibition of amphetamine- and apomorphine-induced behavioural effects by neuropeptide $Y$ Y1 receptor antagonist BIBO 3304. Neuropharmacology. 2000;39:1292-302.
69. Casey DE. Side effect profiles of new antipsychotic agents. J Clin Psychiatry. 1996;57(Suppl 1):40-52.

70. O'keeffe R, Sharman DF, Vogt M. Effect of drugs used in psychoses on cerebral dopamine metabolism. Br J Pharmacol. 1970;38:287-304.

71. Belzung C, Griebel G. Measuring normal and pathological anxiety-like behaviour in mice: a review. Behav Brain Res. 2001;125:141-9.

72. File SE, Hyde JRG. A test of anxiety that distinguishes between the actions of benzodiazepines and those of other minor tranquilisers and of stimulants. Pharmacol Biochem Behav. 1979;11:65-9.

73. Megens AAHP, Awouters FHL, Schotte A, Meert TF, Dugovic C, Niemegeers CJE, et al. Survey on the pharmacodynamics of the new antipsychotic risperidone. Psychopharmacology. 1994;114:9-23.

74. Araseki M, Yamamoto K, Miyashita K. Oxidative stability of polyunsaturated fatty acid in phosphatidylcholine liposomes. Biosci Biotechnol Biochem. 2002;66:2573-7.

\section{Publisher's Note}

Springer Nature remains neutral with regard to jurisdictional claims in published maps and institutional affiliations.
Ready to submit your research? Choose BMC and benefit from:

- fast, convenient online submission

- thorough peer review by experienced researchers in your field

- rapid publication on acceptance

- support for research data, including large and complex data types

- gold Open Access which fosters wider collaboration and increased citations

- maximum visibility for your research: over $100 \mathrm{M}$ website views per year

At BMC, research is always in progress.

Learn more biomedcentral.com/submissions 